\title{
Retrospective study of RAS/PIK3CA/BRAF tumor mutations as predictors of response to first-line chemotherapy with bevacizumab in metastatic colorectal cancer patients
}

\author{
Izuma Nakayama, Eiji Shinozaki , Tomohiro Matsushima, Takeru Wakatsuki, Mariko Ogura, Takashi Ichimura,
} Masato Ozaka, Daisuke Takahari, Mitsukuni Suenaga, Keisho Chin, Nobuyuki Mizunuma and Kensei Yamaguchi

\begin{abstract}
Background: After analysis of minor RAS mutations (KRAS exon 3, 4/NRAS) in the FIRE-3 and PRIME studies, an expanded range of RAS mutations were established as a negative predictive marker for the efficacy of anti-EGFR antibody treatment. BRAF and PIK3CA mutations may be candidate biomarkers for anti-EGFR targeted therapies. However, it remains unknown whether RAS/PIK3CA/BRAF tumor mutations can predict the efficacy of bevacizumab in metastatic colorectal cancer. We assessed whether selection according to RAS/PIK3CA/BRAF mutational status could be beneficial for patients treated with bevacizumab as first-line treatment for metastatic colorectal cancer.

Methods: Of the 1001 consecutive colorectal cancer patients examined for RAS, PIK3CA, and BRAF tumor mutations using a multiplex kit (Luminex ${ }^{\oplus}$ ), we studied 90 patients who received combination chemotherapy with bevacizumab as first-line treatment for metastatic colorectal cancer. The objective response rate (ORR) and progression-free survival (PFS) were evaluated according to mutational status.

Results: The ORR was higher among patients with wild-type tumors (64.3\%) compared to those with tumors that were only wild type with respect to KRAS exon 2 (54.8\%), and the differences in ORR between patients with wild-type and mutant-type tumors were greater when considering only KRAS exon 2 mutations (6.8\%) rather than RAS/PIK3CA/BRAF mutations (18.4\%). There were no statistically significant differences in ORR or PFS between all wild-type tumors and tumors carrying any of the mutations. Multivariate analysis revealed that liver metastasis and RAS and BRAF mutations were independent negative factors for disease progression after first-line treatment with bevacizumab.
\end{abstract}

Conclusions: Patient selection according to RAS/PIK3CA/BRAF mutations could help select patients who will achieve a better response to bevacizumab treatment. We found no clinical benefit of restricting combination therapy with bevacizumab for metastatic colorectal cancer patients with EGFR-wild type tumors.

Keywords: RAS mutation, PIK3CA mutation, BRAF mutation, Colorectal cancer, bevacizumab

\footnotetext{
* Correspondence: eiji.shinozaki@jfcr.or.jp

Department of Gastroenterology, Cancer Institute Hospital of the Japanese

Foundation for Cancer Research, 3-8-31 Ariake, Koto-ku, Tokyo 135-8550,

Japan
} 


\section{Background}

The EGFR signaling pathway has a key role in the proliferation and survival of colorectal cancer cells. Point mutations in exon 2 of the KRAS gene have been shown to be negative predictive markers of the response to anti-EGFR treatment, and consequently anti-EGFR antibodies were not administered to patients with KRAS exon 2 mutant tumors [1]. After a retrospective analysis of minor $R A S$ mutations (e.g. KRAS exon 3 and 4/NRAS) in the FIRE-3 and PRIME studies [2, 3], the so called "all RAS mutation" also came to be regarded as a negative biomarker for anti-EGFR antibody treatment [4]. In addition to $R A S, B R A F$ and PIK3CA mutations are potential biomarkers of response to anti-EGFR targeted therapies [5]. However, it remains unknown whether EGFR pathway mutations affect the efficacy of bevacizumab $(\mathrm{Bmab})$ in metastatic colorectal cancer (mCRC). We evaluated the significance of $R A S / P I K 3 C A / B R A F$ tumor mutations in patients receiving combination chemotherapy with Bmab as the first-line treatment for $\mathrm{mCRC}$, and we assessed whether these mutations could be used to select patients who would derive the greatest clinical benefit from Bmab.

\section{Methods}

\section{Patients}

This was a retrospective study conducted at a single Japanese institute and approved by the ethics committee of Cancer Institute Hospital of Japanese Foundation for Cancer Research (No.2009-1048). Of the 1001 consecutive patients with histologically confirmed CRC who were examined for tumor RAS, PIK3CA, and BRAF mutations in our institute between November 2006 and December 2013, 90 patients were administered combination chemotherapy with Bmab as the first-line treatment for mCRC. Patients who received neo-adjuvant chemotherapy (NAC) or adjuvant chemotherapy completed less than 6 months before enrollment to this study were excluded. Patients who had undergone surgery for metastatic sites were included if it had been performed more than 4 weeks earlier. Patients were required to have adequate hematologic, hepatic, cardiac, and renal function. Their medical records were reviewed to obtain data on clinicopathologic variables. All patients provided written informed consent before receiving treatment.

\section{Procedure}

The treatment regimen was determined by the physician for each patient. The following regimens were employed: modified FOLFOX6 plus Bmab consisted of a fortnightly course of Bmab (5 mg/kg intravenously over 30 to $90 \mathrm{~min}$ on day 1$)$, oxaliplatin $\left(85 \mathrm{mg} / \mathrm{m}^{2}\right.$ intravenously over $2 \mathrm{~h}$ on day 1$)$ plus $1-\mathrm{LV}\left(200 \mathrm{mg} / \mathrm{m}^{2}\right.$ intravenously over $2 \mathrm{~h}$ on day 1$)$ and 5 -fluorouracil (5-FU) $\left(400 \mathrm{mg} / \mathrm{m}^{2}\right.$ bolus on day 1 , followed by infusion of $2400 \mathrm{mg} / \mathrm{m}^{2}$ over $46 \mathrm{~h})$; and CapeOX plus Bmab consisted of oxaliplatin $\left(130 \mathrm{mg} / \mathrm{m}^{2}\right.$ intravenously over $2 \mathrm{~h}$ on day 1 ) plus oral capecitabine $\left(1000 \mathrm{mg} / \mathrm{m}^{2}\right.$ twice daily for 2 weeks in a 3week cycle). Bmab $(7.5 \mathrm{mg} / \mathrm{kg})$ was administered ahead of oxaliplatin intravenously on day 1 every 3 weeks. FOLFIRI plus Bmab consisted of fortnightly courses of Bmab ( $5 \mathrm{mg} / \mathrm{kg}$ intravenously over 30 to $90 \mathrm{~min}$ on day 1 ), irinotecan $\left(150 \mathrm{mg} / \mathrm{m}^{2}\right.$ intravenously over $2 \mathrm{~h}$ on day 1$)$ plus $1-\mathrm{LV}\left(200 \mathrm{mg} / \mathrm{m}^{2}\right.$ intravenously over $2 \mathrm{~h}$ on day 1$)$ and 5 -FU ( $400 \mathrm{mg} / \mathrm{m}^{2}$ bolus on day 1 , followed by infusion of $2400 \mathrm{mg} / \mathrm{m}^{2}$ over $46 \mathrm{~h}$ ).

DNA was extracted from formalin-fixed paraffinembedded (FFPE) tumor tissue, which was mostly obtained at biopsy. Mutations in KRAS codons 12 and 13 were examined using a kit based on a Luminex assay (MEBGEN KRAS Mutation Detection kit, MBL). A Luminex based kit (GENOSEARCH Mu-PACK, MBL) was also used to detect a total of 36 mutations in KRAS (codons 61 and 146), NRAS (codons 12, 13, and 61), PIK3CA (codons 542, 545, 546, and 1047) and BRAF (codon 600). The concordance of findings based on this newly developed multiplex assay kit with conventional direct sequencing results was confirmed previously [6].

\section{Statistical analysis}

The objective response rate (ORR) was evaluated according to the Response Evaluation Criteria in Solid Tumors (RECIST) ver. 1.1. The progression-free survival (PFS) and overall survival (OS) were calculated using the KaplanMeier method. PFS was defined as the duration of survival from the start of chemotherapy to the date of recurrence or death from any cause, whichever occurred first. Patients with no recurrence until the cut-off date were regarded as censored on the last date when no recurrence had been proven by imaging. The disease-progression date was retrospectively re-analyzed by the investigator, and was defined as the date on which progression was first detected using a computed tomography $(\mathrm{CT})$ or fluorodeoxyglucosepositron emission tomography (FDG-PET) scan. If treatments were discontinued before or continued after diseaseprogression due to adverse events or the patient's request, they were censored at the time of the last radiological examination. OS was defined as survival from the start of chemotherapy to death from any cause. For patients who were lost to follow-up, data were censored on the date when the patient was last known to be alive. The data cut-off date was August 12, 2015. A one-sided Fisher's exact test was used to assess the statistical significance of the difference between ORRs according to mutational status at a significance level of $2.5 \%$. Both PFS and OS were estimated using the KaplanMeier method and compared using the log-rank test at a significance level of $5 \%$. In addition to RAS, PIK3CA, and $B R A F$ tumor mutations, variables with a $P$ value less than 
Table 1 Baseline patient characteristics $(n=90)$

\begin{tabular}{|c|c|}
\hline Characteristic & N (\%) \\
\hline \multicolumn{2}{|l|}{ Sex } \\
\hline Male & $48(53.3)$ \\
\hline Female & $42(46.7)$ \\
\hline Median age (range), years & $63(27-79)$ \\
\hline \multicolumn{2}{|l|}{ ECOG performance status } \\
\hline 0 & $82(91.1)$ \\
\hline 1,2 & $8(8.9)$ \\
\hline \multicolumn{2}{|l|}{ Site of primary tumor } \\
\hline RCC (cecum to the transverse colon) & $34(37.8)$ \\
\hline LCRC (descending to the rectosigmoid colon) & $40(44.4)$ \\
\hline Rectum & $16(17.8)$ \\
\hline \multicolumn{2}{|l|}{ Mode of metastasis } \\
\hline Synchronous & $77(85.6)$ \\
\hline Asynchronous & $13(14.4)$ \\
\hline \multicolumn{2}{|l|}{ Sites of metastasis } \\
\hline Liver & 51 \\
\hline Lung & 38 \\
\hline Distant lymph nodes & 29 \\
\hline Peritoneum & 23 \\
\hline \multicolumn{2}{|l|}{ Histology } \\
\hline Differentiated & $83(92.2)$ \\
\hline Undifferentiated & $7(7.8)$ \\
\hline \multicolumn{2}{|l|}{ Number of metastases } \\
\hline 1 & $37(41.1)$ \\
\hline$\geq 2$ & $53(58.9)$ \\
\hline \multicolumn{2}{|l|}{ Chemotherapy regimen } \\
\hline FOLFOX4/mFOLFOX6 or XELOX & $86(95.6)$ \\
\hline FOLFIRI & $4(4.4)$ \\
\hline \multicolumn{2}{|l|}{ Prior metastatectomy } \\
\hline Yes & $12(13.3)$ \\
\hline No & $78(86.7)$ \\
\hline \multicolumn{2}{|l|}{ Resection of primary tumor } \\
\hline Yes & $67(74.4)$ \\
\hline No & $23(25.6)$ \\
\hline \multicolumn{2}{|l|}{ Previous oxaliplatin treatment as adjuvant $C T x$} \\
\hline Yes & $13(14.4)$ \\
\hline No & $77(85.6)$ \\
\hline \multicolumn{2}{|l|}{ KRAS status (codon 12,13) } \\
\hline Wild-type & $47(52.2)$ \\
\hline Mutant & $43(47.8)$ \\
\hline \multicolumn{2}{|l|}{ RAS status (KRAS/NRAS) } \\
\hline Wild-type & $42(46.7)$ \\
\hline Mutant & $48(53.3)$ \\
\hline
\end{tabular}

Table 1 Baseline patient characteristics $(n=90)$ (Continued)

PIK3CA status

Wild-type

$82(91.1)$

Mutant

$8(8.9)$

BRAF status

Wild-type

$82(91.1)$

Mutant

8 (8.9)

ECOG Eastern Cooperative Oncology Group, RCC right-sided colon cancer, $L C R C$ left-sided colorectal cancer, CTx chemotherapy

0.05 in a univariate analysis were included in a multivariate Cox regression analysis. All analyses were performed with EZR (Saitama Medical Center, Jichi Medical University, Saitama, Japan), which is a graphical user interface for $\mathrm{R}$ software (The R Foundation for Statistical Computing) [7].

\section{Results}

Baseline characteristics

The baseline characteristics of the patients are shown in Table 1 . Their median age was 63 years (range, $27-79$ years). Forty-eight patients (53.3\%) were men and 42 patients (46.7\%) were women. Almost all of the subjects had a good performance status. Seventy-four patients $(82.2 \%)$ had colon cancer and $16(17.8 \%)$ had rectal cancer, including right-sided colon cancer (RCC) in 34 patients $(37.8 \%)$ and left-sided colorectal cancer (LCRC) in 56 patients $(62.2 \%)$. RCC was defined as a tumor arising from the cecum to the transverse colon, excluding the appendix, while LCRC was defined as a tumor arising from the descending colon to the rectum. Of these 90 patients, the tumors of 43 patients (46.7\%) were found to have a mutation in KRAS exon 2. In total 48 patients $(53.3 \%)$ had a $R A S$ mutation (KRAS/ NRAS). Seven patients (8.9\%) had a PIK3CA mutation, and another $7(8.9 \%)$ had a $B R A F$ mutation. Thirty-three patients $(36.7 \%)$ had tumors with no RAS, PIK3CA, or BRAF mutation.

\section{Treatment exposure}

Almost all patients received an oxaliplatin-containing regimen, which was FOLFOX in 34 cases $(37.8 \%)$ and XELOX in 52 cases (57.8\%). Among them, 13 (14.4\%) had been administered oxaliplatin prior to this treatment as an adjuvant therapy. The primary tumor was resected in 67 patients $(74.4 \%)$ and 13 patients $(14.4 \%)$ underwent a metastatectomy.

\section{ORR, PFS, and OS}

The median follow-up period for all eligible patients was $23.5(0.8-41.4)$ months, and 51 patients $(56.7 \%)$ died by the cut-off date. Seventy-seven of the 90 patients had measurable lesions. The overall ORR was $52.6 \%$ whilst the ORR of patients with no detected tumor mutations was $64.3 \%$. The ORRs of patients with a PIK3CA or 
$B R A F$ tumor mutation were very low (28.6\%), and more than $40 \%$ of patients with a BRAF tumor mutation had confirmed disease progression at the first evaluation (Fig. 1). Although the ORR varied according to the mutational status of the tumor, these differences were not statistically significant (Table 2). The differences in ORR became gradually greater among patients with wild-type tumors as restricting treatment subjects from only wild type with respect to $K R A S$ exon 2, all $R A S$ wild-type toward all wild-type (RAS/PIK3CA/BRAF). The difference in ORR between the whole population and patients with all wild-type tumors was $11.7 \%$ (Fig. 2).

The overall median OS and PFS were 27.7 and 13.3 months, respectively. The ORR of patients with KRAS exon 2 wild-type and mutant-type tumors differed by $6.2 \%$, and these populations had nearly identical Kaplan-Meier curves for PFS (Fig. 3). The difference between ORRs (18.4\%) was larger when comparing patients with wild-type tumors to those with a tumor carrying any mutation. There was no statistically significant difference in PFS between these groups, although there was a slightly larger difference in Kaplan-Meier curves (Fig. 3).

Univariate analysis revealed that an elevated serum Creactive protein (CRP) level $(>0.05 \mathrm{mg} / \mathrm{dl})$, an unresectable primary tumor, and liver metastases were associated with a significantly shorter PFS (Table 3). Multivariate analysis that included RAS, PIK3CA, and BRAF tumor mutations and baseline prognostic variables revealed that liver metastasis, unresectable primary tumor, $R A S$ and $B R A F$ tumor mutations had independent prognostic value for early progression (Table 3 ).

\section{Discussion}

In clinical practice, it is often not as easy to conduct CT scans at regular intervals as it is in clinical trials. We considered that the ORR would be a relatively rigid parameter for evaluating the efficacy of first-line treatment in clinical practice. In this study, treatment was discontinued due to not only disease progression, but also adverse events or patient refusal, and 21 patients discontinued treatment before disease progression was confirmed by imaging. Time to treatment failure (TTF) is sometimes chosen as an endpoint instead of PFS in clinical trials. However, we considered that PFS would be a more suitable endpoint to evaluate the biological activity of the tumor and drug resistance compared to TTF. OS was the most rigid endpoint but would be determined by not only the first-line treatment but also by second-line and subsequent treatments. The difference in OS may be a result of anti-EGFR therapy after the first-line treatment in patients who have KRAS or RAS wild-type tumors. We therefore assessed the relationship between clinicopathologic factors including RAS, PIK3CA, and BRAF tumor mutation status and PFS.

In this study, we found that patients with a RAS, PIK3CA, or BRAF tumor mutation had a lower ORR than patients with tumors that did not carry these mutations, although this difference was not statistically significant. Patient selection according to tumor mutations in the EGFR pathway might improve the overall response to combination therapy with Bmab as a first-line treatment for mCRC. However, these differences in ORRs could not translate into an improved PFS. Multivariate analysis revealed a negative predictive value of $R A S$ and $B R A F$ tumor mutations with respect to first-line Bmab treatment. In this study, all patients were treated with Bmab, and hence, we could not clarify whether these gene mutations had predictive or prognostic value.

Data from preclinical research has indicated that changes in the EGFR signaling pathway might be related to the efficacy of anti-VEGF therapy [8]. Post-analysis of the AVF2107g trial revealed that adding Bmab to cytotoxic chemotherapy was beneficial regardless of KRAS exon 2 mutation status [9]. KRAS exon 2 mutations are not

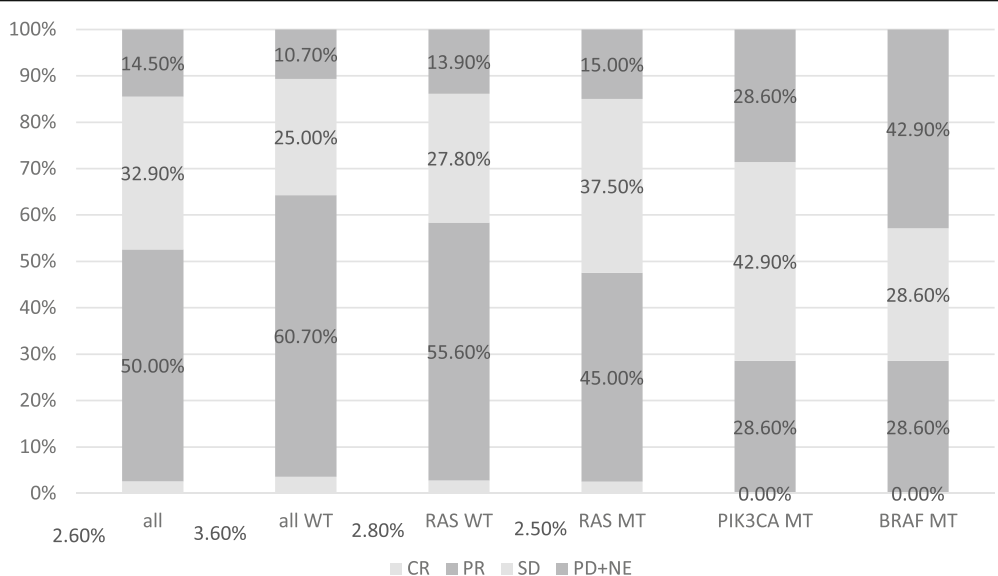

Fig. 1 Response according to tumor mutation status 
Table 2 Response according to mutational status

\begin{tabular}{|c|c|c|c|c|}
\hline & $\begin{array}{l}\text { KRAS exon } 2 \\
\text { wt vs. mt }\end{array}$ & $\begin{array}{l}\text { RAS } \\
\text { wt vs. mt }\end{array}$ & $\begin{array}{l}\text { PIK3CA/BRAF } \\
\text { wt vs. mt }\end{array}$ & $\begin{array}{l}\text { RAS/PIK3CA/BRAF } \\
\text { All wt vs. any } \mathrm{mt}\end{array}$ \\
\hline Responder (n) & 23 vs. 17 & 21 vs. 19 & 38 vs. 2 & 18 vs. 22 \\
\hline Non-responder (n) & 19 vs. 18 & 15 vs. 21 & 31 vs. 5 & 10 vs. 26 \\
\hline ORR (\%) & 54.8 vs. 48.6 & 58.4 vs. 47.5 & 55.1 vs. 28.6 & 64.3 vs. 45.9 \\
\hline Difference in ORR & 6.8 & 10.9 & 26.5 & 18.4 \\
\hline$P$ value ${ }^{*}$ & 0.651 & 0.368 & 0.246 & 0.155 \\
\hline
\end{tabular}

Responder, CR + PR; Non-responder, SD + PD + NE; ORR, overall response rate; wt, wild-type; mt, mutant

${ }^{*}$ calculated using Fisher's exact test

regarded as predictive markers of Bmab treatment. However, we found that the ORR, PFS, and OS differed between patients with KRAS wild-type and mutant tumors (ORR, 60.0\% vs. 41.2\%; PFS, 13.5 months vs. 9.3 months; OS, 27.7 months vs. 19.9 months). These findings are mostly similar to those of other trials comparing clinical outcomes between patients with KRAS exon 2 wild-type and mutant tumors [10-12]. Although statistically significant differences in OS and PFS between patients with KRAS exon 2 wild-type and mutant-type tumors were only shown in the MACRO trial [12], there were numerical differences shown all other trials. KRAS exon 2 wild-type tumors may predict a favorable prognosis. In a retrospective analysis of the PEAK, FIRE-3, and CALGB/SWOG80405 trials, this trend was also apparent when including subjects with any $R A S$ tumor mutation, rather than just those with $K R A S$ exon 2 tumor mutations [13-15]. A recent retrospective analysis of data from the TRIBE trial suggested that tumor mutations in both $B R A F$ and $R A S$ genes predicted a poor outcome for patients undergoing firstline treatment with Bmab plus FOLFIRI or FOLFOXIRI, although $R A S$ mutations had less impact than $B R A F$ mutations [16]. Larger patient numbers would be needed to translate the difference in ORR between patients with wild-type and mutant tumors into an improved clinical outcome, which may be why only the MACRO trial, with the largest number of patients, revealed statistically significant differences in outcome between patients with KRAS wild-type and mutant-type tumors.

Mutations in the BRAF gene have been shown to be markers of a poor prognosis following $\mathrm{mCRC}$ treatment $[17,18]$ and have a stronger prognostic value than $R A S$ mutations [19]. However, $B R A F$ mutant cases were very rare, only 8 in this cohort. It is therefore very difficult to obtain an adequate number of these cases to show statistically significant differences.

Taking into account these previous data, $R A S$ and $B R A F$ mutations may be associated with the inferior efficacy of Bmab treatment. However, due to the relatively small effect of $R A S$ mutations and the rarity of $B R A F$ mutations, we were unable to show statistically significant differences in the ORR and PFS of patients undergoing first-line treatment with Bmab for mCRC.

The ORR and PFS in patients with any of the examined mutations were $45.9 \%$ and 10.8 months, respectively, in this study. These were comparable with those of patients with KRAS exon 2 mutant tumors treated using FOLFOX4 alone in the OPUS study (ORR, 52.0\%; PFS, 8.6 months) [20]. However, in the cetuximab arm of the OPUS and CRYSTAL trials, the ORR and PFS of patients with KRAS

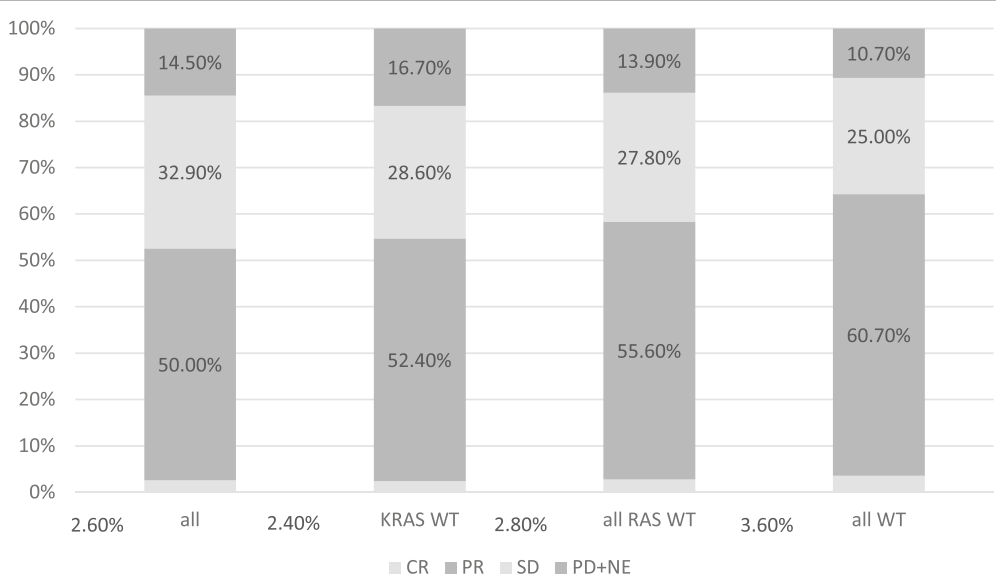

Fig. 2 Responses among patients with wild-type tumor KRAS exon 2, RAS, and RAS/PIK3CA/BRAF 

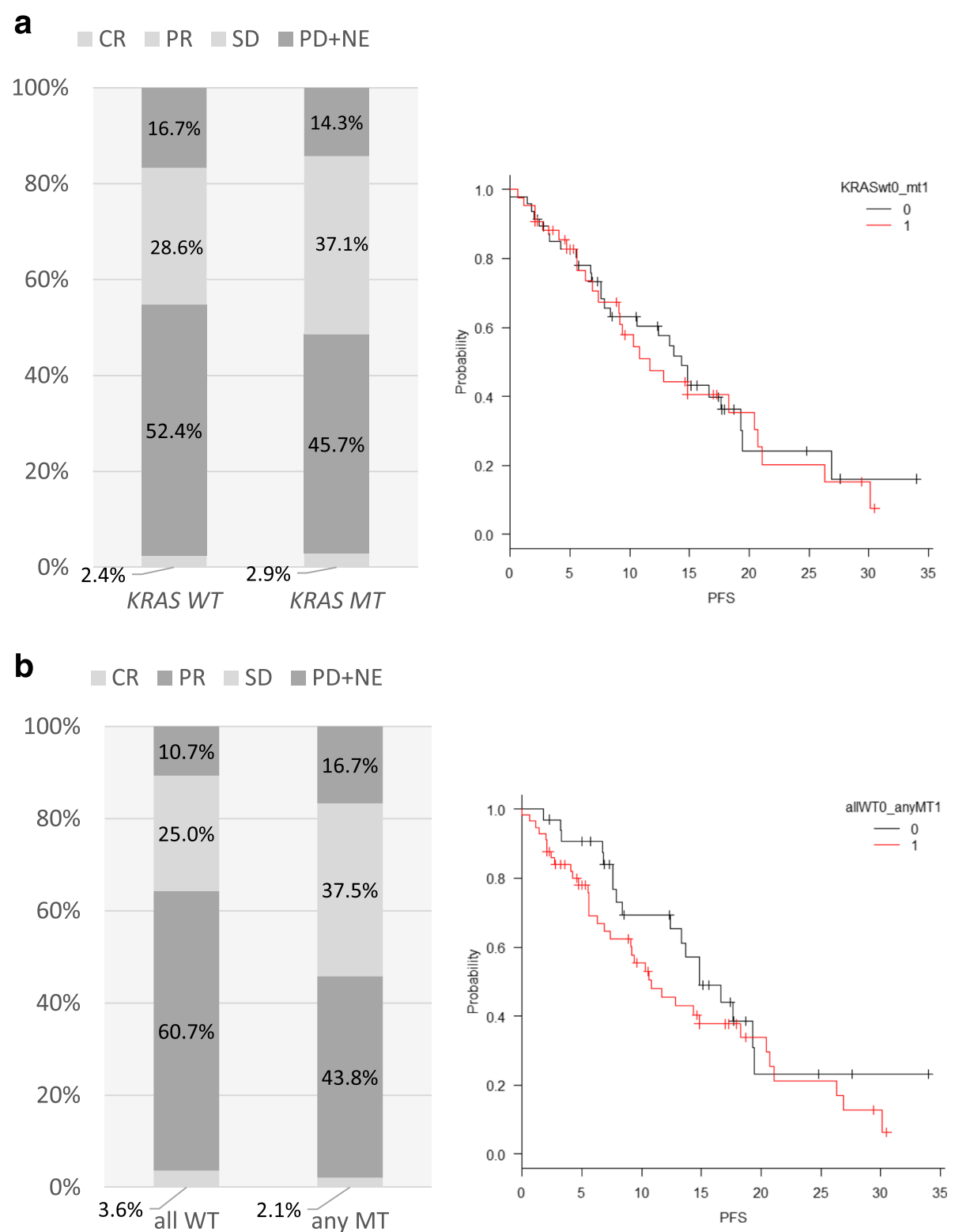

Fig. 3 Relationship between overall response rate (ORR) and progression free survival (PFS) in patients with wild-type or mutant (a) KRAS exon 2 and (b) RAS/PIK3CAVBRAF tumors

mutant tumors were much worse (ORR, 26.0 and 31.3\% respectively; PFS, 5.5 and 7.4 months, respectively) than patients with no tumor mutations in our study [20, 21]. Our study did not show that mCRC patients with a tumor mutation in RAS, PIK3CA, or BRAF had a poorer response to combination chemotherapy with Bmab compared with patients who had none of these mutations. As such, there are insufficient data to justify the exclusion of patients with a RAS, PIK3CA, or BRAF tumor mutation from $B$ mab treatment regimens.

Our study has several limitations. Firstly, this was retrospective cohort study conducted at a single institute. Secondly, there was selection bias in the treatment of patients with $K R A S$ wild-type tumors, especially those who had metastases only in the liver. In our institute, patients with KRAS wild-type tumors and liver only metastases were administered anti-EGFR therapy as an initial standard treatment in order to achieve conversion to metastatectomy. Patients with KRAS wild-type tumors and liver metastases in this cohort had relatively unfavorable factors, such as a high tumor burden or multiple organ metastases, and patients with relatively favorable factors were usually excluded. This may explain why liver metastasis was an independent predictor of a poor prognosis in this cohort. Patients with RAS wild-type tumors in this cohort might have had a poor prognosis, 
Table 3 Univariate and multivariate analysis of pr4gression-free survival (PFS) $(n=90)$

\begin{tabular}{|c|c|c|c|c|}
\hline \multirow[t]{2}{*}{ Variable } & \multicolumn{2}{|l|}{ Univariate analysis } & \multicolumn{2}{|l|}{ Multivariate analysis } \\
\hline & $\mathrm{HR}(95 \% \mathrm{Cl})$ & $P$ value & $\mathrm{HR}(95 \% \mathrm{Cl})$ & $P$ value \\
\hline \multicolumn{5}{|l|}{ Sex } \\
\hline Male vs. female & 1.0 vs. $0.72(0.42-1.24)$ & 0.236 & & \\
\hline \multicolumn{5}{|l|}{ Age } \\
\hline$<63$ y.o vs. $\geq 63$ y.o & 1.0 vs. $0.82(0.48-1.40)$ & 0.472 & & \\
\hline \multicolumn{5}{|l|}{ ECOG PS } \\
\hline 0 vs. 1,2 & 1.0 vs. $0.66(0.21-2.14)$ & 0.490 & & \\
\hline \multicolumn{5}{|l|}{ Site of primary tumor } \\
\hline RCC vs. LCRC & 1.0 vs. $0.88(0.51-1.52)$ & 0.643 & & \\
\hline \multicolumn{5}{|l|}{ Differentiated-type } \\
\hline yes vs. no & 1.0 vs. $1.78(0.75-4.22)$ & 0.188 & & \\
\hline \multicolumn{5}{|l|}{ Synchronous mets } \\
\hline yes vs. no & 1.0 vs. $0.61(0.29-1.30)$ & 0.201 & & \\
\hline \multicolumn{5}{|l|}{ Sites of metastasis } \\
\hline Non-liver vs. liver & 1.0 vs. $1.84(1.06-3.20)$ & 0.031 & 1.0 vs. $3.26(1.57-6.77)$ & 0.002 \\
\hline Non-lung vs. lung & 1.0 vs. $1.17(0.68-2.00)$ & 0.567 & & \\
\hline Non-LN vs. LN & 1.0 vs. $0.67(0.37-1.20)$ & 0.177 & & \\
\hline Non-P vs. $P$ & 1.0 vs. $0.95(0.51-1.78)$ & 0.878 & & \\
\hline \multicolumn{5}{|l|}{ Number of metastases } \\
\hline 1 vs. $\geq 2$ & 1.0 vs. $0.87(0.51-1.50)$ & 0.628 & & \\
\hline \multicolumn{5}{|l|}{ Primary resection } \\
\hline yes vs. no & 1.0 vs. $2.00(1.09-3.66)$ & 0.024 & 1.0 vs. $2.13(1.05-4.29)$ & 0.035 \\
\hline \multicolumn{5}{|l|}{ Prior L-OHP } \\
\hline yes vs. no & 1.0 vs. $0.75(0.35-1.60)$ & 0.457 & & \\
\hline \multicolumn{5}{|l|}{$\operatorname{ALP}(/)$} \\
\hline$\leq$ ULN Vs. $>$ ULN & 1.0 vs. $1.07(0.56-2.04)$ & 0.836 & & \\
\hline \multicolumn{5}{|l|}{ LDH (/IU) } \\
\hline$\leq$ ULN Vs. $>$ ULN & 1.0 vs. $1.27(0.74-2.21)$ & 0.387 & & \\
\hline \multicolumn{5}{|l|}{ CRP (mg/dl) } \\
\hline$\leq$ ULN Vs. $>$ ULN & 1.0 vs. $2.35(1.32-4.17)$ & $<0.001$ & 1.0 vs. $1.57(0.79-3.10)$ & 0.196 \\
\hline \multicolumn{5}{|l|}{ CEA } \\
\hline$\leq U L N$ vs. $>$ ULN & 1.0 vs. $0.90(0.49-1.65)$ & 0.735 & & \\
\hline \multicolumn{5}{|l|}{ CA19-9 } \\
\hline$\leq$ ULN Vs. $>$ ULN & 1.0 vs. $1.63(0.95-2.80)$ & 0.076 & & \\
\hline \multicolumn{5}{|l|}{ RAS status } \\
\hline Wild-type vs. mutant & 1.0 vs. $1.36(0.79-2.32)$ & 0.264 & 1.0 vs. $2.01(1.07-3.76)$ & 0.030 \\
\hline \multicolumn{5}{|l|}{ PIK3CA status } \\
\hline Wild-type vs. mutant & 1.0 vs. $1.00(0.36-2.77)$ & 0.993 & 1.0 vs. $0.66(0.21-2.03)$ & 0.466 \\
\hline \multicolumn{5}{|l|}{ BRAF status } \\
\hline Wild-type vs. mutant & 1.0 vs. $1.60(0.68-3.75)$ & 0.285 & 1.0 vs. 3.87 (1.38-10.9) & 0.010 \\
\hline
\end{tabular}

$C I$ confidence interval, HR hazard ratio, RCC right-sided colon cancer, $L C R C$ left-sided colorectal cancer, mets metastasis, $L N$ lymph node, $P$ peritoneum, ULN upper limit of normal, NA not assessable. All data in italics are with $p$-value $<0.05$

compared to those included in other studies, and this selection bias might have affected the outcome of patients with tumors that do not carry mutations in the genes studied here. Thirdly, due to the rare incidence of PIK3CA and $B R A F$ mutation in CRC, we could evaluate only small number patients with these mutations. 


\section{Conclusion}

There were no statistically significant differences in ORR and PFS according to mutations in EGFR pathway genes in patients receiving cytotoxic chemotherapy with Bmab as the first-line treatment for mCRC. RAS/PIK3CA/ $B R A F$ mutations could help identify tumors that will respond to both anti-EGFR antibodies and Bmab. However, this study did not find a clinical benefit for restricting Bmab treatment to $\mathrm{mCRC}$ patients with tumors that have wild-type EGFR pathway genes.

\section{Additional file}

Additional file 1: Row data about clinicopathological features and clinical outcomes with omitting personally identifiable information of patients. (XLSX $27 \mathrm{~kb})$

\section{Abbreviations}

5-FU: 5-fluorouracil; Bmab: bevacizumab; CT: Computed tomography; EGFR: Epidermal growth factor receptor; FFPE: Formalin-fixed paraffin-embedded; LCRC: Left-sided colon; mCRC: Metastatic colorectal cancer; NAC: Neo-adjuvant chemotherapy; ORR: Objective response rate; OS: Overall survival; PET: Positron emission tomography; PFS: Progression free survival; RCC: Right-sided colon; RECIST: Response Evaluation Criteria in Solid Tumors; TTF: Time to treatment failure

\section{Acknowledgments}

The authors would like to thank the staff who managed patients at the ambulatory treatment center and on the ward. We also thank Editage (www.editage.jp) for English language editing.

\section{Fundings}

There is no funding to be declared for publication in this article.

\section{Availability of data and materials}

All data generated or analyzed during this study are included in this published article and its Additional file 1.

\section{Authors' contributions}

IN analyzed the clinical data and wrote the original manuscript. IN, ES, TM, TW, MO, TI, MO, DT, MS, KC, NM and KY were all involved in the administration of chemotherapy. All authors contributed to editing the manuscript and approved the final version.

\section{Competing interests}

Eiji Shinozaki: Honoria from Merck Serono Co.,Ltd, Takeda Co.,Ltd, Brystol Myers Squibb Japan Co.,Ltd, Taiho Co.,Ltd, Chugai Co., Ltd, Ono Co,.Ltd The remaining authors declare that they have no competing interests.

\section{Consent for publication}

Not applicable.

\section{Ethics approval and consent to participate}

The present study was conducted according to the principles of the declaration of Helsinki and all participating patients provided written informed consent. This study was approved by the ethics committee of Cancer Institute Hospital of Japanese Foundation for Cancer Research (No.2009-1048).

Received: 26 December 2015 Accepted: 13 December 2016 Published online: 09 January 2017

\section{References}

1. Douillard JY, Oliner KS, Siena S, Tabernero J, Burkes R, Barugel M, et al. K-ras Mutations and Benefit from Cetuximab in Advanced Colorectal Cancer. N Engl J Med. 2013;369:1023-34.

2. Heinemann V, von Weikersthal LF, Decker T, Kiani A, Vehling-Kaiser U, Al-Batran SE, et al. FOLFIRI plus cetuximab versus FOLFIRI plus bevacizumab as first-line treatment for patients with metastatic colorectal cancer (FIRE-3): a randomised, open-label, phase 3 trial. Lancet Oncol. 2014;15:1065-75.

3. Douillard JY, Oliner KS, Siena S, Tabernero J, Burkes R, Barugel M, et al. Panitumumab-FOLFOX4 treatment and RAS mutations in colorectal cancer. N Engl J Med. 2013;369:1023-34.

4. Sorich MJ, Wiese MD, Rowland A, Kichenadasse G, McKinnon RA, Karapetis CS, et al. Extended RAS mutations and anti-EGFR monoclonal antibody survival benefit in metastatic colorectal cancer: a meta-analysis of randomized, controlled trials. Ann Oncol. 2015;26:13-21.

5. De Roock W, De Vriendt V, Normanno N, Ciardiello F, Tejpar S. KRAS, BRAF, PIK3CA, and PTEN mutations: implications for targeted therapies in metastatic colorectal cancer. Lancet Oncol. 2011;12:594-603.

6. Bando H, Yoshino T, Shinozaki E, Nishina T, Yamazaki K, Yamaguchi K, et al. Simultaneous identification of 36 mutations in KRAS codons 61 and 146, BRAF, NRAS, and PIK3CA in a single reaction by multiplex assay kit. BMC Cancer. 2013;13:405

7. Kanda Y. Investigation of the freely available easy-to-use software 'EZR' for medical statistics. Bone Marrow Transplant. 2013;48:452-8.

8. Ince WL, Jubb AM, Holden SN, Holmgren EB, Tobin P, Sridhar M, et al. Association of k-ras, b-raf, and p53 Status With the Treatment Effect of bevacizumab. J Natl Cancer Inst. 2005;97:981-9.

9. Hurwitz HI, Yi J, Ince W, Novotny WF, Rosen O. The clinical benefit of bevacizumab in metastatic colorectal cancer is independent of $\mathrm{K}$-ras mutation status: analysis of a phase III study of bevacizumab with chemotherapy in previously untreated metastatic colorectal cancer. Oncologist. 2009;14:22-8

10. Hecht JR, Mitchell E, Chidiac T, Scroggin C, Hagenstad C, Spigel D, et al. A randomized phase IIIB trial of chemotherapy, bevacizumab, and panitumumab compared with chemotherapy and bevacizumab alone for metastatic colorectal cancer. J Clin Oncol. 2009;27:672-80.

11. Price TJ, Hardingham JE, Lee CK, Weickhardt A, Townsend AR, Wrin JW, et al. Impact of KRAS and BRAF Gene Mutation Status on Outcomes From the Phase III AGITG MAX Trial of Capecitabine Alone or in Combination With Bevacizumab and Mitomycin in Advanced Colorectal Cancer. J Clin Oncol. 2011;29:2675-82.

12. Díaz-Rubio E, Gómez-España A, Massutí B, Sastre J, Reboredo M, Manzano JL, et al. Role of Kras status in patients with metastatic colorectal cancer receiving first-line chemotherapy plus bevacizumab: a TTD group cooperative study. PLoS One. 2012;7:e47345.

13. Karthaus M. Updated Overall Survival Analysis of Novel Predictive KRAS /NRAS Mutations beyond KRAS exon 2 in PEAK: A 1st-line Phase 2 Study of FOLFOX6 plus Panitumumab or Bevacizumab in Metastatic Colorectal Cancer. Poster session presented at: The European Cancer Congress 2013. 38 ${ }^{\text {th }}$ annual conference of the European Society for Medical Oncology; 201327 Sep-01 Oct: Amsterdam, Netherlands.

14. Heinemann $V$, et al. Analysis of KRAS /NRAS and BRAF Mutations in FIRE-3: A Randomized Phase III Study of FOLFIRI plus Cetuximab or Bevacizumab as First-line Treatment for Wild-type KRAS (exon 2) Metastatic Colorectal Cancer Patients. Poster session presented at: The European Cancer Congress 2013. $38^{\text {th }}$ annual conference of the European Society for Medical Oncology; 2013 27 Sep - 01 Oct: Amsterdam, Netherlands.

15. Lenz HJ, et al. CALGB/SWOG 80405: Phase III Trial of Irinotecan/5-FU/ Leucovorin (FOLFIRI) or Oxaliplatin/5-FU/Leucovorin (mFOLFOX6) with Bevacizumab (BV) or Cetuximab (CET) for Patients (Pts) with Untreated Metastatic Adenocarcinoma of the Colon or Rectum (MCRC): Expanded RAS analyses. Poster session presented at: The European Cancer Congress 2014. $39^{\text {th }}$ annual conference of the European Society for Medical Oncology; 2014 26 Sep-30 Sep: Madrid, Spain.

16. Cremolini C, Loupakis F, Antoniotti C, Lupi C, Sensi E, Lonardi S, et al. FOLFOXIRI plus bevacizumab versus FOLFIRI plus bevacizumab as first-line treatment of patients with metastatic colorectal cancer: updated overall survival and molecular subgroup analyses of the open-label, phase 3 TRIBE study. Lancet Oncol. 2015;16:1306-15.

17. Brookmeyer C, Van Cutsem E, Rougier P, Ciardiello F, Heeger S, Schlichting M, et al. Addition of cetuximab to chemotherapy as first-line treatment for KRAS wild-type metastatic colorectal cancer: pooled analysis of the CRYSTAL and OPUS randomised clinical trials. Eur J Cancer. 2012;48:1466-75.

18. Tie J, Gibbs P, Lipton L, Christie M, Jorissen RN, Burgess AW, et al. Optimizing targeted therapeutic development: analysis of a colorectal cancer patient population with the BRAF (V600E) mutation. Int J Cancer. 2011;128:2075-84 
19. Richman SD, Seymour MT, Chambers P, Elliott F, Daly CL, Meade AM, et al. KRAS and BRAF mutations in advanced colorectal cancer are associated with poor prognosis but do not preclude benefit from oxaliplatin or irinotecan: results from the MRC FOCUS trial. J Clin Oncol. 2009:27:5931-7.

20. Bokemeyer C, Bondarenko I, Hartmann JT, de Braud F, Schuch G, Zubel A, et al. Efficacy according to biomarker status of cetuximab plus FOLFOX-4 as first-line treatment for metastatic colorectal cancer: the OPUS study. Ann Oncol. 2011;22:1535-46.

21. Van Cutsem E, Köhne CH, Láng I, Folprecht G, Nowacki MP, Cascinu S, et al. Cetuximab plus irinotecan, fluorouracil, and leucovorin as first-line treatment for metastatic colorectal cancer: updated analysis of overall survival according to tumor KRAS and BRAF mutation status. J Clin Oncol. 2011;29:2011-9.

Submit your next manuscript to BioMed Central and we will help you at every step:

- We accept pre-submission inquiries

- Our selector tool helps you to find the most relevant journal

- We provide round the clock customer support

- Convenient online submission

- Thorough peer review

- Inclusion in PubMed and all major indexing services

- Maximum visibility for your research

Submit your manuscript at www.biomedcentral.com/submit 